\section{SMAD men}

\section{By Tim Fulmer, Senior Writer}

University of Colorado Denver School of Medicine researchers have shown that topical application of the signaling protein Smad7 can treat and prevent oral mucositis in mice. ${ }^{1}$ The team will now test the compound in mice with cancer-associated mucositis-a condition with limited therapeutic options.

Oral mucositis can occur in a variety of clinical settings. In particular, it is a serious side effect in about $80 \%$ of patients with head and neck cancer receiving high-dose radiotherapy. ${ }^{2}$ It causes severe pain and can force patients to use a feeding tube and ventilation apparatus, and it may lead to serious infections in mucosal sores and ulcerations.

Standard of care is a combination of scrupulous oral hygiene and antibiotics. The only targeted oral mucositis drug is Kepivance palifermin, a recombinant keratinocyte growth factor (KGF) marketed by Swedish Orphan Biovitrum AB to treat oral mucositis in patients with blood cancer receiving radiation before bone marrow transplants.

However, the drug has shown only modest clinical benefit in two investigator-run head and neck cancer trials, in which it failed to significantly decrease the incidence of oral mucositis compared with placebo. ${ }^{3,4}$

Because excessive inflammation and destruction of epithelial cells are the hallmarks of oral mucositis, the Colorado team hypothesized that local delivery of an anti-inflammatory with the additional ability to promote growth of oral epithelial cells might help treat the condition in patients with cancer.

Indeed, the group's prior work in mice showed that the endogenous intracellular signaling protein SMAD7 (mothers against decapentaplegic homolog 7; MADH7) both antagonized proinflammatory signaling pathways and blocked apoptosis of keratinocytes in the oral epithelia. ${ }^{5-7}$ The open question was whether a recombinant form of SMAD7 could be delivered directly to the oral mucosa and generate a therapeutic effect.

To confirm that high Smad7 levels reduced the damaging effects of radiation on the oral mucosa, the researchers first irradiated transgenic mice overexpressing Smad7 and compared them with irradiated wildtype mice.

The Smad7 animals were more resistant to radiation-induced oral mucositis and had significantly lower levels of multiple inflammation markers in the oral cavity, including leukocyte infiltration $(p<0.001)$, epithelial cell apoptosis $(p<0.001)$, NF- $\kappa \mathrm{B}$ expression $(p<0.001)$ and transforming growth factor- $\beta$ (TGFB; TGF $\beta$ ) signaling $(p<0.05)$.

The researchers next looked for similar effects when they delivered Smad7 directly to the oral epithelia of irradiated wild-type mice. To do that, they generated a recombinant form of Smad7 and linked it to the Tat protein, which allowed Smad7 to cross the cell membrane and enter the nucleus of epithelial cells.

In mice, daily application of the Smad7-Tat protein fusion to the oral cavity 24 hours before irradiation significantly decreased ulcer formation compared with saline control application $(p<0.01)$. The fusion's effects were comparable to those of Kepivance. Immunostaining confirmed that Smad7-Tat significantly reduced apoptotic cells and NF- $\kappa \mathrm{B}$ signaling in the oral mucosa $(p<0.01)$.

Finally, in irradiated mice with existing oral mucositis, Smad7-Tat led to smaller ulcers and lower mucosal pathology than saline control, confirming that the compound had both a therapeutic and a preventative effect.

The study was published in Nature Medicine. It was led by Qinghong Zhang, assistant professor of dermatology at the University of Colorado School of Medicine, and Xiao-Jing Wang, professor and director of the Head, Neck and Skin Cancer Research Program at the university.

The researchers now will test Smad7 delivery in irradiated cancer models, Wang told SciBX. In addition, the group is "designing and testing different Smad7 constructs and production systems to optimize its largescale production and purification so that it can be safely used in humans and also meet potential market demands," she said.

The findings are covered by patent applications and available for partnering or licensing from the University of Colorado Denver School of Medicine, said Wang.

Fulmer, T. SciBX 6(12); doi:10.1038/scibx.2013.278

Published online March 28, 2013

\section{REFERENCES}

1. Han, G. et al. Nat. Med;; published online March 10, 2013; doi: $10.1038 / \mathrm{nm} .3118$

Contact: Xiao-Jing Wang, University of Colorado Denver School of Medicine, Aurora, Colo.

e-mail: xj.wang@ucdenver.edu

Contact: Qinghong Zhang, same affiliation as above e-mail: qinghong.zhang@ucdenver.edu

2. Conway, D.I. Evid. Based Dent. 7, 81-82 (2006)

3. Le, Q.-T. et al. J. Clin. Oncol. 29, 2808-2814 (2011)

4. Henke, M. et al. J. Clin. Oncol. 29, 2815-2820 (2011)

5. Hong, S. et al. Nat. Immunol. 8, 504-513 (2007)

6. Han, G. et al. Dev. Cell 11, 301-312 (2006)

7. He, W. et al. EMBO J. 21, 2580-2590 (2002)

COMPANIES AND INSTITUTIONS MENTIONED

Swedish Orphan Biovitrum AB (SSE:SOBI), Stockholm, Sweden University of Colorado Denver School of Medicine, Aurora, Colo. 